\title{
La ciudad cerrada y su diversificación como reto del Área Metropolitana de Guadalajara, México'
}

\author{
Bernd Pfannenstein², Edgar Eduardo Anacleto Herrera ${ }^{3}$ \\ y Salvador Sevilla Villalobos ${ }^{4}$
}

\begin{abstract}
RESUMEN
El fenómeno de las urbanizaciones cerradas se convirtió en un objeto dinámico y complejo que se ha estado transformando durante las últimas décadas, logrando un cambio similar en las ciudades latinoamericanas donde se encuentra. El Área Metropolitana de Guadalajara, México, no ha sido la excepción y padece la re-transformación del concepto. El "éxito" alcanzado por las urbanizaciones cerradas en Guadalajara llevó a la inclusión de nuevos estratos económicos en este esquema de vivienda fortificada, una inserción que se traduce en el $15 \%$ de la ciudad construida bajo la modalidad de espacio de acceso limitado gracias a la imposición de barreras físicas que fragmentan el territorio. Mediante la construcción de una base de datos georreferenciada se ha logrado un análisis preliminar del fenómeno en esta metrópolis mexicana, arrojando resultados que constatan las modificaciones conceptuales que diversos investigadores identificaron y que están causando una estratificación del modelo de vivienda.
\end{abstract}

Palabras clave: Ciudad cerrada, urbanizaciones cerradas, segregación, planificación urbana, Guadalajara.

\begin{abstract}
The phenomenon of gated communities has evolved into a dynamic and complex object that has transformed Latin American Cities over the last decades. The Metropolitan Area of Guadalajara, Mexico, is also undergoing a retransformation of this concept. The "success" of gated residential areas in Guadalajara has been defined by the inclusion of a new economic strata into this fortified housing scheme. Fifteen percent of the built city are characterized by the modality of limited access due to physical barriers that have caused a fragmentation of this territory. Through the implementation of a geo-referenced database, a spatial analysis of the phenomenon has been accomplished in this Mexican city, showing results that confirm the conceptual modifications that several researchers have identified and that are causing a stratification of this housing model.
\end{abstract}

Key words: Gated city, gated communities, segregation, urban planning, Guadalajara.

El autor principal agradece al Instituto Metropolitano de Planeación del Área Metropolitana de Guadalajara (IMEPLAN) la colaboración académica llevada a cabo en el marco de los términos de referencia estabilizados para la construcción de la base de datos - (TDR_SIM_I, proyecto 2, 2014-2015) y al programa "Innovative Governance of Large Urban Systems" de la Escuela Politécnica Federal de Lausana, Suiza, por su colaboración en el seguimiento del mismo proyecto.-. Artículo recibido el 20 de abril de 2016, aceptado el 15 de septiembre de 2016 y corregido 23 de abril de 2017.

Universidad de Passau (Alemania). E-mail: b.pfannenstein@outlook.com

Universidad de Guadalajara (México). E-mail: e_anacleto@outlook.com

Universidad de Guadalajara (México). E-mail: s.sevilla@outlook.com 
Desde hace algunas décadas es posible apreciar en Latinoamérica la expansión de un esquema de urbanización, conocido en el ámbito anglosajón cómo "gated communities" (Becerril-Sánchez et al., 2013: 192), que ha desencadenado una serie de procesos que están reorganizando la estructura y funcionalidad de los territorios, caracterizándose por ser un modelo de vivienda que repercute directamente en la percepción de la ciudad como lugar continuo, habitable y de bien común, ya que produce una denigración del espacio urbano debido a la imposición de barreras físicas que fragmentan la interacción social.

También llamadas "barrios cerrados", "condominios fechados" o "countries", las urbanizaciones cerradas residenciales responden a un área construida de tipo habitacional donde se ha restringido el acceso mediante un cierre perimetral que cuenta con un mecanismo de control de paso, son un prototipo de ciudad que se fundamenta en la privatización del espacio público como garantía de tranquilidad en la vida cotidiana (Enríquez, 2006).

El fenómeno de las urbanizaciones cerradas ha logrado tener una presencia de escala mundial gracias a que opera sobre un marco económico y socio-cultural que se ampara en el capitalismo global (Prada, 2011) por lo que, en la actualidad se puede apreciar un fuerte desarrollo de vivienda amurallada en las principales ciudades de varios países importantes de América Latina como Chile (Borsdorf e Hidalgo, 2004; Hidalgo, 2004; Hidalgo et al., 2003), Argentina (Arizaga, 2003; Carballo, 2002; Thuillier, 2005), Brasil (De Lima \& Ribeiro 2002; Souza, 2008) y México (Enríquez, 2007; Becerril-Sánchez et al., 2013; Cabrales y Canosa, 2001, 2002; Rodríguez y Mollá, 2003), espacios donde los diferentes estudios realizados constatan las modificaciones que se están dando en las estructuras urbanas y sociales.

Por sus atributos, autores como Díaz y Ortíz (2013) hacen una comparativa entre estos espacios habitacionales y los antiguos bastiones medievales puesto que funcionan de manera similar, permitiendo el establecimiento de sistemas de control, disuasión, coerción, invisibilidad, blindaje, inaccesibilidad y vigilancia, aspectos que hoy en día sirven para potencializar los ideales de seguridad, exclusividad, privacidad y plusvalía sobre los que se realiza la promoción de las urbanizaciones cerradas (Ickx, 2002).

La ciudad cerrada pudiera no ser considerada una problemática per se debido a que la gente que vive en ella ha decidido auto segregarse para intentar remediar una serie de deficiencias que se perciben en el medio urbano (Roitman, 2004) sin embargo, cuando la cantidad de unidades y el área que ocupan representan una proporción importante de la superficie urbanizada, se vuelve discutible el impacto que logra su concentración y la exclusión de la población en general como consecuencia de decisiones tomadas por unos cuantos individuos. Es clara la afectación que sufre la ciudadanía, sobre todo tras limitarse su accesibilidad urbana y fortalecerse el patrón de desconexión territorial, efectos que se generan a raíz de la estructura de ciudad segregada y dividida que propicia la urbanización cerrada y su particular patrón de asentamiento insular que forma una imagen de archipiélago de islas dentro de un océano que se vería representado por la ciudad abierta (Hidalgo et al., 2003; Janoschka, 2002).

La situación del Área Metropolitana de Guadalajara -AMG-, en México, es de resaltarse pues se estima que más del 14 por ciento de la ciudad está bajo este concepto de fraccionamientos 
cerrados debiéndose subrayar casos particulares de municipios integrantes de la metrópolis que alcanzan una proporción de superficie urbana intramuros mayor al 30 por ciento.

Ante la cuestionable situación de un territorio donde casi la tercera parte de la superficie construida se encuentra dividida por una barda perimetral, es necesario preguntarse, ¿las urbanizaciones cerradas son el modelo correcto para afrontar la carencia técnica y operacional que la administración pública tiene en el cumplimiento de su deber con la ciudadanía? o si por el contrario, ¿son solo un producto de consumo exitoso que fue bien recibido por las poblaciones latinoamericanas de altos recursos económicos?; un resultado que está inmerso dentro del marco de renovados ordenes neoliberales que se configura a partir de ideologías ancladas en la modernidad latinoamericana (Romo, 2015).

\section{Una perspectiva sobre el discurso de las urbanizaciones cerradas}

Si bien es cierto que los orígenes y las problemáticas que se asocian a la presencia de urbanizaciones cerradas suelen ser similares a escala global, son un producto derivado del desarrollo de políticas neoliberales aplicadas al territorio, y del mismo modo en que estas tienen un carácter geográficamente variable por las dificultades que implica trasladarlas sin que se relacionen con las condiciones concretas del lugar donde se aplican (Hidalgo y Janoschka, 2014), la reproducción de urbanizaciones cerradas en diversos países se ve influenciada por características específicas de cada sitio y generan un amplio marco para el análisis (Prada, 2011).

Roitman (2004) hace una agrupación de las diferentes circunstancias que, desde su perspectiva, indujeron la aparición del esquema habitacional cerrado mediante dos categorías: causas de carácter estructural y causas relacionadas con la acción social. En la primera clase se encuentran factores como el aumento de inseguridad, temor a hechos delictivos, el fracaso que sufre el Estado como proveedor de servicios públicos, el crecimiento en la brecha social entre ricos y pobres, y la polarización entre grupos; en la segunda, se contemplan aspectos que conciernen a la acción social encaminada a lograr un mejor estilo de vida, evitar los problemas urbanos como la pobreza y la búsqueda de homogeneidad social.

De manera similar, Vidal-Koppmann (2014) atribuye el origen de la urbanización cerrada al desarrollo de prácticas sociales de integración y/o segregación de ciertos estratos sociales, la creación de muros como respuesta al incremento de la criminalidad y por último, como parte de un proceso de fragmentación y ruptura del orden espacial. Estos argumentos están altamente relacionados con los expuestos por Prada (2011), a diferencia de que este último, habla sobre un auge vivido dentro del modelo del fraccionamiento de acceso restringido a raíz de la presencia de cuatro elementos explicativos fundamentales: postmodernidad, fragmentación urbana, miedo e inversiones en valores inmobiliarios.

Otros autores que han trabajado el tema desde una perspectiva general tienen conclusiones similares respecto a los componentes que participaron en la expansión significativa de las urbanizaciones cerradas en Latinoamérica (Cabrales, 2005; Díaz y Ortíz, 2013; Riwilis, 2008), pero 
ya revisando casos específicos, se detectará que según el ámbito donde se desarrollaron se da mayor relevancia a ciertas causas.

En el escenario mexicano se destacan como principales factores de éxito la incapacidad del Estado para garantizar los derechos ciudadanos básicos y el creciente problema de inseguridad (Cabrales, 2005), contexto en el que sobresalen dos conceptos que permiten entender el funcionamiento de los fraccionamientos cerrados en Guadalajara: seguridad y segregación.

La importancia del primer factor se da a partir de la relación que hay en la dicotomía seguridad-inseguridad y la percepción psicológica que emana de ella y se representa a través del sentimiento del miedo - individual o colectivo-, un efecto que tiene fuerte influencia en el desarrollo urbano y es reconocido como elemento que acompaña la evolución morfológica de las ciudades (Villar \& García, 2016).

Diferentes trabajos subrayan la relevancia que tiene esta percepción como pieza clave en el éxito de la urbanización cerrada en América Latina (Arizaga, 2003; Cabrales, 2005; Enríquez, 2006; Hidalgo, 2004; Malizia y Paolasso, 2009; Roitman, 2004), y aunque los índices de criminalidad han evolucionado desfavorablemente (Montesino, 2010), queda en duda si realmente el aumento ha sido tan elevado o más bien se trata de una idea equívoca resultado de los métodos empleados por los medios de comunicación para abordar el tema (Borsdorf e Hidalgo, 2004), una deficiencia que ha sido aprovechada como "argumento de marketing" para lograr un aumento en la demanda de urbanizaciones de acceso privado ante la idea de vivir en una ciudad segura (Vidal-Koppmann, 2014).

Sea real o no el incremento delincuencial que muestran los medios, este mecanismo para ofrecer seguridad, al igual que en otras ciudades, está fortaleciendo la aceptación del modelo provocando una modificación en los roles y funciones de los actores y agentes urbanos involucrados en la producción de ciudad a través de la implementación de políticas neoliberales (Romo, 2015). Una serie de acciones que llevan a una "división social del espacio" (Schteingart, 2001: 18) y dan cabida al segundo concepto, la segregación como acción social que es motivada directamente por el miedo (Villar \& García, 2016).

No solo es relevante por ser un producto del entorno latinoamericano inseguro, sino que la segregación social, o diferenciación entre sectores de la población, contribuye en la explicación de las transformaciones socio-espaciales que están logrando las urbanizaciones cerradas en los principales centros urbanos (Becerril-Sánchez et al., 2013). Algo que es importante señalar y en lo que Borsdorf (2002) pone especial atención, es que esta perspectiva no tiene fundamento suficiente para explicar y evaluar el fenómeno completo, esto debido a que siempre ha habido una diferenciación socio-espacial en el entorno urbano relacionada con la clara estratificación social que posee, pero ante la llegada de las comunidades amuralladas, se manifiesta como un nuevo esquema para el que no existe una teoría general que permita su estudio.

Hablar únicamente de segregación es referirse a un mecanismo de reproducción de desigualdad social (Méndez y Ariel, 2008), distinción en las posibilidades que tiene la población para acceder a componentes importantes de la ciudad como áreas verdes y espacios públicos (Pérez, 2013), además de una interposición de distancia entre los estratos sociales (Linares y Lan, 2007), 
pero para poder analizar el cambio conceptual que se origina por la llegada de la ciudad fortificada, Roitman (2004) propone sustentar el análisis en la teoría estructuralista de Anthony Giddens y así poder explicar parte fundamental de este nuevo proceso de segregación urbana que es generada por la interposición de barreras físicas, la asociación voluntario-involuntario.

Al entenderse esta teoría como las condiciones que rigen la continuidad y modificación de las estructuras y por ende de los sistemas, se puede analizar la forma en que "el sistema social afecta la acción individual y a través del cual la acción individual afecta al sistema social" (ibídem: 13). Visto en la práctica, la teoría permite analizar cómo es que las contrariedades que persisten en la ciudad orillaron al individuo a buscar nuevas alternativas para satisfacer sus necesidades, encontrando una solución provisional en la ciudad amurallada, aceptando su desarrollo; una decisión que termina transformando el sistema social y afectando a la ciudadanía como conjunto.

La retroalimentación del fenómeno es constante y lo encamina hacia una significativa "re-ocupación, re-apropiación y re-organización de los espacios vividos" (Martínez, 2001: 78) mediante la migración de las elites a zonas rurales periféricas, motivados por la búsqueda espacios de mayor tamaño, calidad y seguridad. Este proceso fue posible gracias a los atributos de control que otorga el fraccionamiento cerrado y que permiten la convivencia cercana con la población de bajos recursos económicos que originalmente ocupaba el área (Camus, 2015; Díaz \& Ortiz, 2013; Hidalgo et al., 2008).

El uso de términos discriminatorios como "los otros" (Roitman, 2004: 10), puede ser considerado parte del producto surgido de una aproximación física entre grupos a causa de la suburbanización de las élites (Sabatini et al., 2012), un hecho que desencadenó en una serie de factores que resaltan aún más la división social que hay en la ciudad de Guadalajara, sobre todo porque el modelo habitacional terminó atrayendo a sectores de población que, según el esquema original de urbanización cerrada, deberían ser parte de la población que se encuentra fuera del muro y no de la que pertenece al estilo de vida fortificado.

Este nuevo panorama, donde se detectan ciertas modificaciones en los conceptos que tradicionalmente están vinculados a la urbanización cerrada, lleva a plantear la necesidad de abordar el caso del Área Metropolitana de Guadalajara y su amurallamiento residencial desde una perspectiva distinta, dejar de lado la idea que consideraba solo a la población de altos recursos económicos como únicos ocupantes del fraccionamiento cerrado, para tomar un enfoque que contemple una diversidad de características económicas y sociales (Borsdorf, 2002; Cabrales, 2006); pasar de una visión que percibe la segregación socio-espacial como parte de una relación afuera/adentro (Roitman, 2004; Riwilis, 2008; Vidal-Koppmann, 2014; Schteingart, 2001) a otra que distinga entre distintas tipologías de urbanizaciones cerradas y su distribución territorial.

\section{Guadalajara: una nueva tipología de urbanizaciones cerradas}

La ciudad de Guadalajara, conformada por la capital del estado de Jalisco y los municipios de Zapopan, San Pedro Tlaquepaque, Tonalá, Tlajomulco de Zúñiga, El Salto, Juanacatlán, Ixtlahuacán de los Membrillos y Zapotlanejo (IMEPLAN, 2015), siendo una de las tres metrópolis más importantes del país (Aguilar y Vázquez, 2000), ha padecido los efectos de la globalización 
y las políticas neoliberales, teniendo como resultado un crecimiento considerable de la mancha urbana gracias a la aplicación de modelos de desarrollo basados en la apertura económica y competencia, así como por la llegada de inversión extranjera que fortaleció la actividad regional (Arias, 1997; Cabrales y Canosa, 2002), un proceso que atrajo gradualmente nuevos esquemas de producción de vivienda para satisfacer la demanda de espacios habitacionales que exigía la población, siendo uno de los principales el de las urbanizaciones cerradas.

Tanto la suburbanización y el encapsulamiento que se describen en Argentina (Arizaga, 2003), así como, la segmentación del espacio público en Chile (Hidalgo et al., 2003) y la importancia del papel que juega la inseguridad en la aceptación de vivienda cerrada que vuelve inconexo el espacio en Colombia (García y Peralta, 2014), son secuelas que están dejando los fraccionamientos cerrados en el AMG al igual que en otros ejemplos mexicanos como Monterrey, Puebla, Toluca y la Ciudad de México (Becerril-Sánchez et al., 2013).

Desde el año de 1967, fecha en la cual se comienza la construcción del primer par de conjuntos habitacionales cerrados, hasta mediados de la década de 1990 se enumeraba en Guadalajara un total de 20 urbanizaciones cerradas destinadas a población de altos recursos económicos (Cabrales y Canosa, 2002), una cantidad que se incrementó rápidamente hacia el 2000 llegando a 150 unidades (Ickx, 2002).

Durante los siguientes años se produjo una expansión considerable en el número de urbanizaciones después de que los diferentes grupos sociales comenzaron a tomar este tipo de vivienda como alternativa principal para garantizar su seguridad (Rodríguez y Mollá, 2003), logrando hasta finales de 2015 una cifra de más de 2.700 unidades habitacionales de diferentes escalas y estratos sociales en los nueve municipios del área metropolitana, crecimiento que se vio favorecido por la endeble y cambiante normatividad en temas de ordenamiento territorial en la que hasta la fecha, no se incluye una definición clara del concepto de "urbanización cerrada".

Posiblemente la reforma que tuvo mayor impacto se da en la Ley General de Asentamientos Humanos posterior a la reestructuración que se hace en 1993 de las atribuciones que tenían el poder federal, estatal y municipal en la administración del suelo urbano. Esta modificación, además de hacer un trasvase de responsabilidades hacia los gobiernos locales, buscó promover la participación privada al permitir "la coordinación y la concentración de acciones e inversiones entre el sector público, en sus tres niveles de gobierno, con el sector privado", generándose así mecanismos e instrumentos financieros, simplificación de trámites para la ejecución de acciones de desarrollo urbanístico, entre otras condiciones que facilitaron la intervención de la iniciativa privada en la gestión del territorio (Ordóñez, 2016).

El aumento y aceptación de políticas neoliberales que llevaron al predominio de la razón económica sobre la política (Ornelas, 2000), junto a la autonomía que el artículo 115 de la Constitución Política Mexicana otorga a cada municipio al brindarle facultades plenas para controlar el proceso de planeación de su territorio (Cámara de Diputados, 2016), crearon un modelo de producción de ciudad enfocado en la rentabilidad del suelo, esquema caracterizado por dar preferencia a proyectos urbanos con posibilidades de generar una mayor ganancia sobre aquellos que priorizan el ordenamiento territorial (Valenzuela, 2002). 
Al igual que como sucedió en otras latitudes, se dio paso a la desregularización del mercado del suelo y se flexibilizaron los instrumentos de planificación territorial en favor de los grandes inversores (Borsdorf e Hidalgo, 2004), desafortunadamente como señalan González y Medina (2010), citando a García, el hecho de dar participación a las constructoras en todos los niveles de financiamiento, producción y comercialización de bienes inmuebles, es considerado como uno de los agentes principales para favorecer intereses particulares.

Las constantes facilidades otorgadas a la iniciativa privada y los cambios sufridos en el ámbito urbano hicieron posible que la unidad habitacional de acceso restringido se colocara como principal oferta inmobiliaria, causando una trasformación paulatina del esquema de vivienda como parte de un efecto asociado a la violencia, inseguridad y tendencia hacia la polarización social, suscitándose un cambio conceptual y estructural del modelo donde ya no solo la gente de altos recursos económicos accedía a él.

Presentado como "tipología de barrios cerrados en América Latina", Borsdorf (2002: 593-600) agrupa a las urbanizaciones cerradas en función de la diversidad de características surgidas del cambio conceptual: barrios cerrados lifestyle, barrio cerrado arribista, barrios cerrados de clase media y barrio cerrado subvencionado; siendo esta última una categoría para los fraccionamientos cerrados ofrecidos a población de bajos recursos como vivienda de interés social contrapuesto al esquema de lujo que caracterizaba a la urbanización cerrada.

Guadalajara participa de este cambio, dejando reflejo de ello en los trabajos que han sido publicados sobre la metrópolis. Hacia finales de 1980 y principios de 1990 el amurallamiento residencial colectivo era visto como "ícono urbano del privilegio y un buen símbolo de poder" (Camus, 2015: 14), perspectiva que es empleada por Cabrales y Canosa (2001) así como por Ickx (2002) en sus estudios, pero en años posteriores, se puede encontrar una propuesta que plantea cambiar la perspectiva de análisis de la urbanización cerrada en Guadalajara con la definición de nuevas tipologías que permitan incluir los fraccionamientos cerrados de altas densidades dirigidos a la clase media-baja (Cabrales, 2006).

La respuesta que tuvo el sector inmobiliario frente a la necesidad de diferentes sectores económicos de adquirir un hogar protegido dentro de un contexto urbano poco seguro y fuertemente degradado fue la causante de la pérdida de atributos asociados al gran estilo de vida y el prestigio social que eran característicos de la urbanización cerrada (Prada, 2011), provocando la aparición de lo que Roitman (2016: 16) identifica como "falsas urbanizaciones cerradas", un intento por recrear las "urbanizaciones cerradas reales" al disponer de cercos, muros perimetrales y rejas, pero que se distinguen por la poca o nula infraestructura interna, la falta de seguridad privada y una notable disminución en el tamaño de los lotes y vivienda.

Tanto la propuesta de Cabrales (2006) como la realizada por Borsdorf (2002) dan lugar para el análisis de las estructuras derivadas de las transformaciones que sufrieron las urbanizaciones cerradas y que son descritas por Roitman (2016) como falsificaciones, sin embargo, posterior a una primera exploración del área de trabajo bajo estos enfoques, se decidió optar por diseñar una clasificación distinta de los fraccionamientos cerrados en Guadalajara que permitiera consolidar las tres metodologías y destacar los hallazgos encontrados. 
Pese al cambio que se ha dado en la conceptualización de la urbanización cerrada, estas estructuras aún mantienen su atributo principal, la capacidad para favorecer la ruptura del continuo urbano al limitar el acceso público físicamente (Enríquez, 2006), por lo tanto, para llevar a cabo la revisión de imágenes satelitales con el fin de delimitar los polígonos habitacionales, se definió la presencia de una barrera física - puede ser un muro, una reja, malla perimetral, etc.- como parámetro "indispensable" para identificar los fraccionamientos cerrados del AMG.

Por otra parte, la presencia de una caseta de vigilancia o algún otro tipo de control de acceso se estableció como un elemento "no indispensable" y así evitar descartar polígonos que caen dentro del esquema de falsa urbanización cerrada, pudiéndose detectar de este modo aquellas estructuras que, aunque no contaban con este aditamento, contribuyen en la división del continuo urbano; la urbanización semi-cerrada.

Esta categoría se definió como una agrupación que dos sub-clases en función de los ejemplos encontrados ya que su origen es distinto pero con implicaciones territoriales similares: El primer conjunto es el de aquellos fraccionamientos cerrados que se "abrieron" por diversas circunstancias; en algunos casos fue por la falta de recursos económicos para cubrir los gastos de seguridad privada y saldar las cuotas de mantenimiento que requiere vivir aislados, en otros se tuvo que permitir el libre tránsito a causa de la presión social del entorno inmediato que demandaba accesibilidad, mientras que unos más eliminaron la restricción de acceso y algunos muros por priorizar la comodidad de los desplazamientos cotidianos ante el sentimiento de inseguridad. Aunque son secciones de la traza urbana donde la posibilidad de entrar ya no está limitada en su totalidad, su estructura de ciudad amurallada persiste en un cierto grado, razón por la cual mantienen su atributo principal como objeto fragmentador del espacio.

El segundo elemento que integra las urbanizaciones semi-cerradas son aquellas áreas que surgieron de manera inversa al ser planeadas como entornos habitacionales que podían cerrarse, pero, donde aún no se logra la restricción de paso. Son espacios donde la configuración de las casas funge como barrera física entre el resto de la ciudad y "el interior", se caracterizan por contar con equipamientos a los que solo se puede acceder mediante una calle principal y algunos cuantos callejones, mismos que pueden ser cerrados con facilidad al colocar un cancel o una valla una vez que se decida solicitar la limitación de paso como vialidad de uso exclusivo.

También se agregó una categoría adicional para clasificar aquellos espacios habitacionales de acceso restringido de pequeña escala y que también pueden entrar como un tipo de falsificación, las micro urbanizaciones cerradas. Estas estructuras se diferencian del resto de urbanizaciones cerradas por su tamaño, poco número de casas y la casi nula presencia de equipamientos y servicios, además de que están ubicados principalmente en zonas urbanas consolidadas; se aprovechan las calles de poca longitud para agrupar las viviendas como parte de una unidad habitacional donde se limita el acceso, y aunque no tienen el mismo impacto de una macro urbanización cerrada, son capaces de generar condiciones similares al resto de fraccionamientos cerrados, el desgaste de la ciudadanía y de su interacción social (Urquieta, 2011: 20).

En el Cuadro $N^{\circ} 1-$ se han organizado las propuestas de Roitman (2016), Cabrales (2006) y Borsdorf (2002) junto con la clasificación propuesta a modo de cuadro comparativo para permitir un análisis entre ellas y lograr un mejor reconocimiento de las relaciones que se pueden encon- 


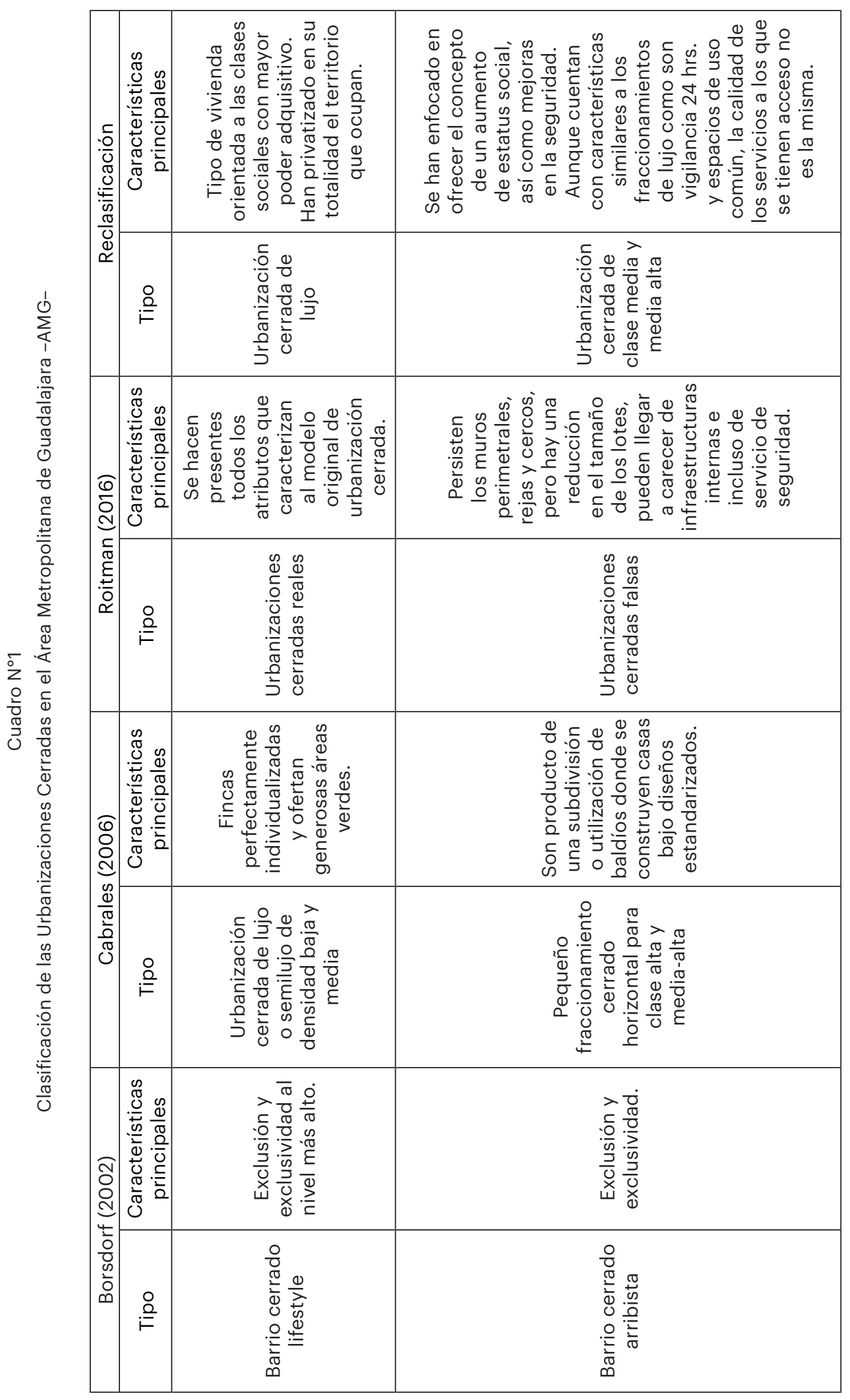




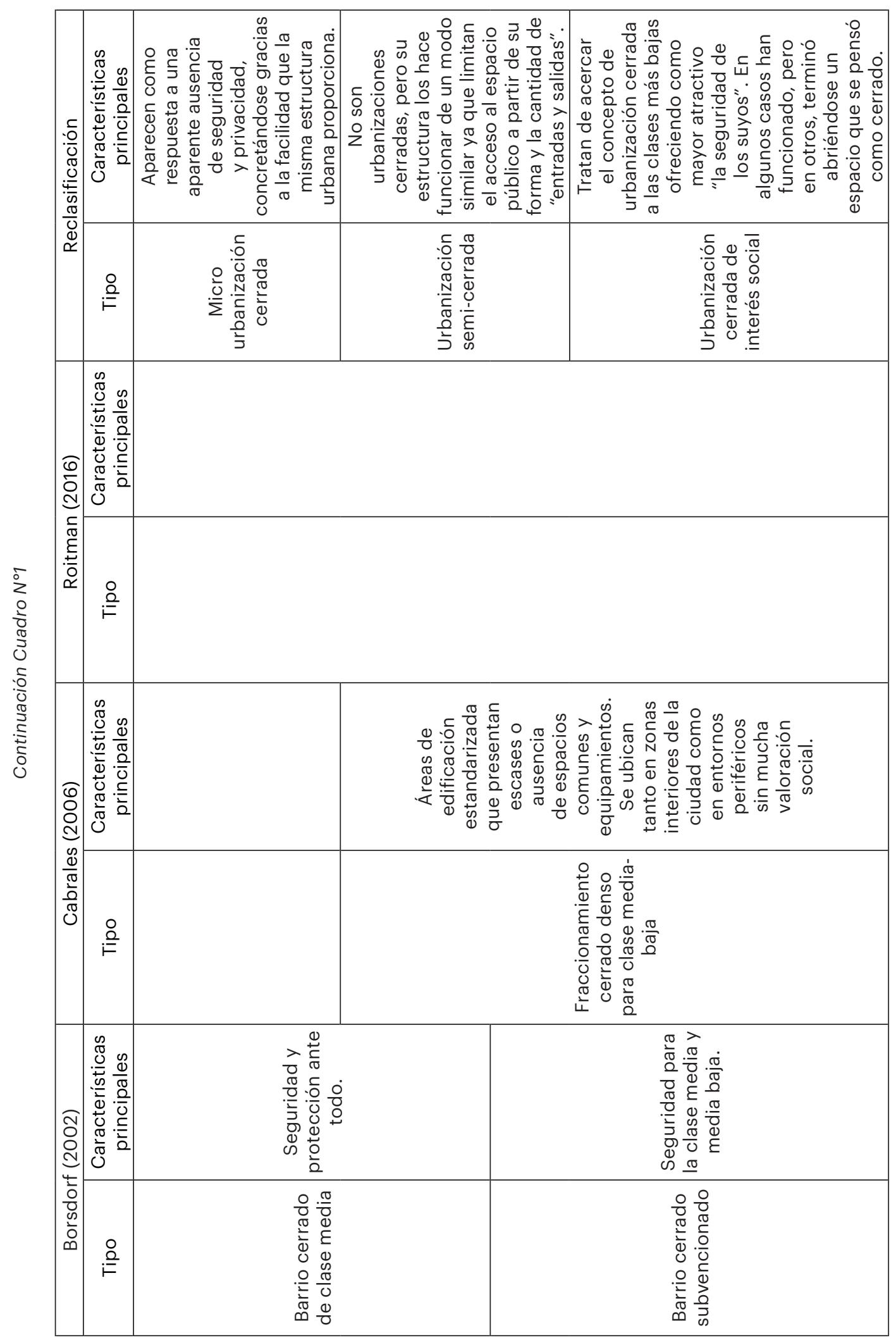

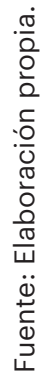


trar entre las características que agrupa cada una, creando un marco de referencia general en el proceso de clasificación de las urbanizaciones cerradas en el Área Metropolitana de Guadalajara.

La intención principal de hacer esta reclasificación de las tipologías de urbanizaciones cerradas es permitir una distinción entre los diferentes grupos de estructuras habitacionales en formato horizontal con las que, mediante la presencia de una barrera física, se ha fragmentado el espacio público al restringir el acceso vehicular y peatonal de manera total o parcial, ello como parte de los atributos principales del concepto de urbanización cerrada (Roitman, 2004), un elemento de la estructura urbana capaz de producir un cambio en el patrón tradicional de segregación (Hidalgo et al., 2003) como consecuencia de la diversificación del concepto y la inclusión de nuevos estratos sociales.

\section{Segregación intramuros. La estratificación de las urbanizaciones cerradas en el Área Metropolitana de Guadalajara}

Para entender mejor la problemática, así como la expansión territorial que ha tenido el fenómeno, es necesario que el primer acercamiento se haga desde una perspectiva general mediante la revisión de los datos absolutos obtenidos de la revisión de imágenes satelitales. Con un total de 2.722 unidades habitacionales, se calcula que existen 10.169,44 hectáreas de superficie urbanizada donde el acceso a la ciudad está restringido o se ve dificultado gracias a la presencia de muros, rejas y otros aditamentos que emplea la urbanización cerrada para aislar a la población que ha decidido auto-segregarse-Cuadro $\mathrm{N}^{\circ} 2-$.

Cuadro $\mathrm{N}^{\circ} 2$

Urbanizaciones Cerradas en el Área Metropolitana de Guadalajara -AMG-, Año 2015

\begin{tabular}{|l|r|r|r|r|}
\hline Municipio & \multicolumn{1}{|c|}{$\begin{array}{c}\text { Área } \\
\text { Urbanizada } \\
\text { (ha) }\end{array}$} & $\begin{array}{c}\text { Cantidad de } \\
\text { Urbanizaciones } \\
\text { Cerradas por } \\
\text { municipio }\end{array}$ & $\begin{array}{c}\text { Urbanizaciones } \\
\text { Cerradas } \\
\text { (ha) }\end{array}$ & $\begin{array}{c}\text { Área } \\
\text { superficie urbanizada } \\
\text { municipal que } \\
\text { se encuentra en } \\
\text { Urbanización Cerrada } \\
\text { (\%) }\end{array}$ \\
\hline El Salto & $3.584,52$ & 5 & 129,16 & $3,60 \%$ \\
\hline Guadalajara & $14.282,87$ & 448 & 215,27 & 1,51 \\
\hline $\begin{array}{l}\text { Ixtlahuacán de los } \\
\text { Membrillos }\end{array}$ & $1.413,27$ & 9 & 179,90 & 12,73 \\
\hline Juanacatlán & 371,38 & 3 & 75,72 & 20,39 \\
\hline San Pedro & $8.715,51$ & 365 & 704,37 & 8,08 \\
\hline Tlaquepaque & $10.626,74$ & 401 & $3.565,35$ & 10,59 \\
\hline Tlajomulco de Zúñiga & $6.829,34$ & 125 & 723,20 & 20,83 \\
\hline Tonalá & $21.834,28$ & 1.361 & $4.548,31$ & 2,38 \\
\hline Zapopan & $1.183,78$ & 5 & 28,16 & 14,77 \\
\hline Zapotlanejo & $68.841,69$ & 2.722 & $10.169,44$ & \\
\hline TOTAL & & & &
\end{tabular}

Fuente: Elaboración propia. 
Aunque en distinta proporción, se puede encontrar urbanizaciones cerradas en todos los municipios del AMG, siendo Zapopan y Tlajomulco de Zúñiga los más afectados debido a la gran cantidad de superficie que ocupa este esquema de vivienda. En el caso del primer municipio la construcción de Valle Real y Puerta de Hierro hacia finales de la década de 1980 (Cabrales y Canosa, 2002) propició un aumento en el número de fraccionamientos cerrados que poco a poco se adueñaron del territorio, mientras que Tlajomulco, además de experimentar un proceso similar con el emplazamiento de El Palomar y Club de Golf Santa Anita hacia finales de la década de 1960 y principios de 1970 (ibídem), el bajo costo del suelo tuvo un papel determinante en la proliferación de fraccionamientos cerrados (Núñez, 2011).

Desde una perspectiva metropolitana es fácil identificar la relevancia que tuvieron estos fraccionamientos cerrados en la definición del rumbo que tomaría el desarrollo del fenómeno, -Figura $\mathrm{N}^{\circ} 1-$, marcando una evidente tendencia periférica con una cargada distribución al lado poniente de la ciudad, manifestando además la participación que tuvieron como agente dispersor de la mancha urbana gracias a su peculiar patrón de crecimiento en forma de "tentáculos" a lo largo de las avenidas principales, caminos rurales y carreteras (González y Medina, 2010: 3).

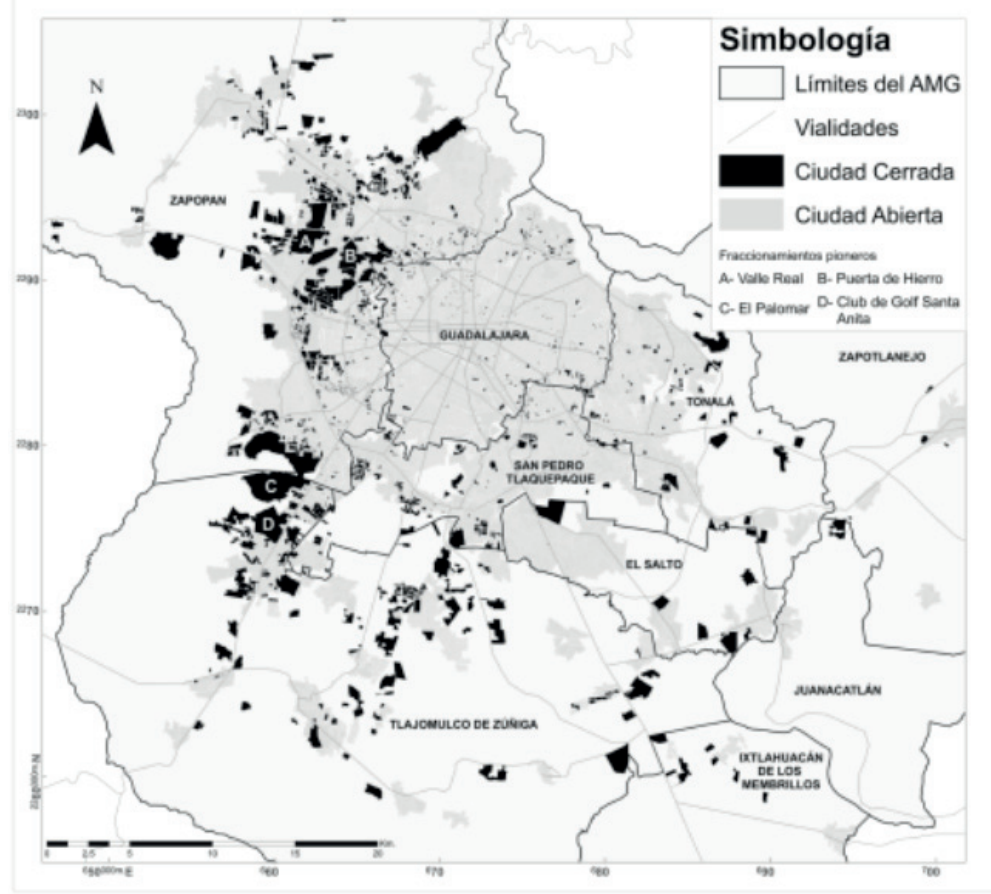

Fuente: Elaboración propia.

La mayoría de problemáticas relacionadas con los fraccionamientos que se pueden encontrar en Guadalajara coinciden con aquellas que se han dado en otros casos latinoamericanos, con la 
salvedad de ciertas diferencias relacionadas con características particulares del territorio, quedando expuesto el gran reto que tiene el Área Metropolitana de Guadalajara de cara al proceso de exclusión espacial que padece, sobre todo por el cambio sustancial que vive el paradigma de la urbanización cerrada y la necesidad por abordar de manera distinta las modificaciones que está logrando la vivienda fortificada en el patrón tradicional de segregación (Hidalgo et al., 2003). Ante la adversidad, se debe plantear un análisis desde el cual se puedan obtener datos que demuestren como cada tipo de fraccionamiento cerrado re-estructura la ciudad en función de sus características particulares, pudiéndose disminuir de este modo los efectos que ha demostrado las urbanizaciones cerradas en el territorio.

Es importante trabajar este enfoque ya que factores como distancia económica, hablando en términos de la diferencia que existe entre la capacidad adquisitiva de los ciudadanos que viven a cada lado del muro, problemas de movilidad, apropiación del espacio público, fragmentación del territorio, entre otros conflictos, son diferenciados según el sector de la ciudad que se observe, reforzando el planteamiento de que no todos las consecuencias imputadas a la urbanización cerrada como modelo de vivienda son replicados de manera homogénea por cada unidad habitacional, lo que significaría un tratamiento distinto de cada caso específico.

Aunque los valores obtenidos a partir de la categorización de los fraccionamientos cerrados en el Área Metropolitana de Guadalajara -Cuadro $N^{\circ} 3-$ no tienen por si solos la capacidad para dar respuesta al problema general debido a la influencia que tienen otras variables que intervienen en el sistema urbano, pueden resaltar varios puntos importantes surgidos a partir de la interpretación de los resultados, determinando los posibles rumbos de investigaciones futuras gracias a la nueva perspectiva del fenómeno que se logra al estratificar el modelo de urbanización cerrada y destacar sus particularidades.

Cuadro $\mathrm{N}^{\circ} 3$

Número de Urbanizaciones Cerradas según su categoría en el Área Metropolitana de Guadalajara -AMG-, Año 2015

\begin{tabular}{|l|r|r|r|r|}
\hline Categoría & $\begin{array}{c}\text { Número de } \\
\text { Urbanizaciones } \\
\text { Cerradas }\end{array}$ & $\begin{array}{c}\text { Porcentaje } \\
\text { por } \\
\text { categoría }\end{array}$ & $\begin{array}{c}\text { Superficie } \\
\text { comprendida } \\
\text { (ha) }\end{array}$ & $\begin{array}{c}\text { Porcentaje } \\
\text { por } \\
\text { categoría }\end{array}$ \\
\hline Urbanización cerrada de lujo & 233 & 8,56 & $4.289,93$ & 42,18 \\
\hline $\begin{array}{l}\text { Urbanización cerrada de clase } \\
\text { media y media alta }\end{array}$ & 1.553 & 57,05 & $2.122,17$ & 20,87 \\
\hline Micro urbanización cerrada & 618 & 22,70 & 161,43 & 1,59 \\
\hline Urbanización semi-cerrada & 128 & 4,70 & $1.851,94$ & 18,21 \\
\hline $\begin{array}{l}\text { Urbanización cerrada de interés } \\
\text { social }\end{array}$ & 190 & 6,98 & $1.743,98$ & 17,15 \\
\hline
\end{tabular}

Fuente: Elaboración propia.

Lo primero que sobresale es el peso que han alcanzado las categorías que difieren del esquema original de la urbanización cerrada ante el cambio del concepto tradicional, mostrando el 
camino que tomó la vivienda fortificada en el caso particular de Guadalajara, así como el cambio de paradigma que ha sido referido en el trabajo de los autores citados. Hubo una integración de bajos recursos, definiéndose un contraste social entre unidades habitacionales.

Parte del imaginario creado en torno a la urbanización cerrada gira alrededor a las alternativas que puede ofrecer el fraccionamiento cerrado para facilitar el acceso a equipamientos y servicios como casa club, grandes áreas verdes, espacios deportivos, etc; elementos que serán puestos a exclusiva disposición de los residentes del fraccionamiento cerrado, pero, frente la realidad que manifiestan los datos, hay un cierto grado de incertidumbre respecto a la calidad de estos espacios de recreación, e incluso hacia la existencia de los mismos.

El $8,56 \%$ de las unidades encontradas pertenece al sector de lujo y ocupa el $42,18 \%$ de superficie fortificada mientras que el $86,73 \%$ de fraccionamientos cerrados apenas tiene el $39,61 \%$ de cobertura territorial, prácticamente la mitad del espacio amurallado está ocupado solo por el 10\% de unidades. Hay que aclarar que esta afirmación no considera los datos concernientes a fraccionamientos semi-cerrados, ello por tratarse de una categoría que está conformada por unidades habitacionales que son dirigidas tanto al sector de lujo como a sectores de menor poder adquisitivo, habiendo una heterogeneidad de características que deben tratarse de forma distinta.

Los porcentajes reflejan las condiciones en las que se conformaron la mayor parte de urbanizaciones cerradas de "no lujo", ya que de las 2.361 unidades que integran las categorías de urbanizaciones cerradas de clase media y media alta, micro-urbanizaciones cerradas y urbanizaciones cerradas de interés social, 2.023 están definidas por un polígono menor a dos hectáreas de superficie, un estrato de vivienda fortificada de alta densidad que, audazmente, han sido equiparado con los beneficios y calidad de vida que se pueden encontrar en un fraccionamiento de 470 hectáreas; la superficie que alcanza el complejo de lujo con mayor cobertura territorial.

Un segundo punto que debe analizarse a profundidad es el peso real de cada uno de los fraccionamientos cerrados como elemento individual que forma parte de un sistema mayor y la presión que ejercen como objetos en el entorno. Se debe identificar qué problemáticas están ligadas a características específicas de una tipología urbanización cerrada, por ejemplo, la gran extensión de ciertas unidades o una alta concentración de urbanizaciones cerradas de pequeña escala.

Los conflictos viales son la problemática ideal para representar las diferenciaciones a las que se trata de hacer alusión pues, aunque un fraccionamiento de gran envergadura implica un gran número de coches que sobrecargan una calle en horas pico, los pequeños espacios habitacionales cerrados generan presión de manera distinta. Por lo general una urbanización cerrada grande cuenta con uno o dos puntos de entrada y salida de vehículos que están espaciados entre sí, siendo estos los únicos nodos conflictivos en el sistema vial; por su parte, los fraccionamientos de menor tamaño tienen la capacidad de colocarse uno muy cerca del otro gracias a la poca superficie que ocupan, teniendo de este modo la posibilidad de producir una saturación de accesos en una misma vialidad con la subsecuente interrupción del flujo vehicular para permitir la incorporación de los automóviles que intentan salir del fraccionamiento cerrado.

Visto desde el patrón de distribución que muestran las distintas categorías de urbanización cerrada, también se puede inferir que es posible encontrar una diferenciación en el grado de fragmentación socio-espacial vinculado a las particularidades sociales de la población que reside en ella y el lugar donde fue ubicada. La siguiente cartografía -Figura $N^{\circ} 2-$ se diseñó a partir de una 
malla hexagonal en la que a cada polígono se le asignó un valor determinado en función de la proporción de superficie de acceso restringido que contiene por tipología de urbanización cerrada, mostrando de esta manera una mejor perspectiva del grado de concentración de cada categoría.

Figura $\mathrm{N}^{\circ} 2$

Distribución espacial del Grado de Marginación y las tipologías de Urbanizaciones Cerradas en el Área Metropolitana de Guadalajara -AMG-, Año 2015

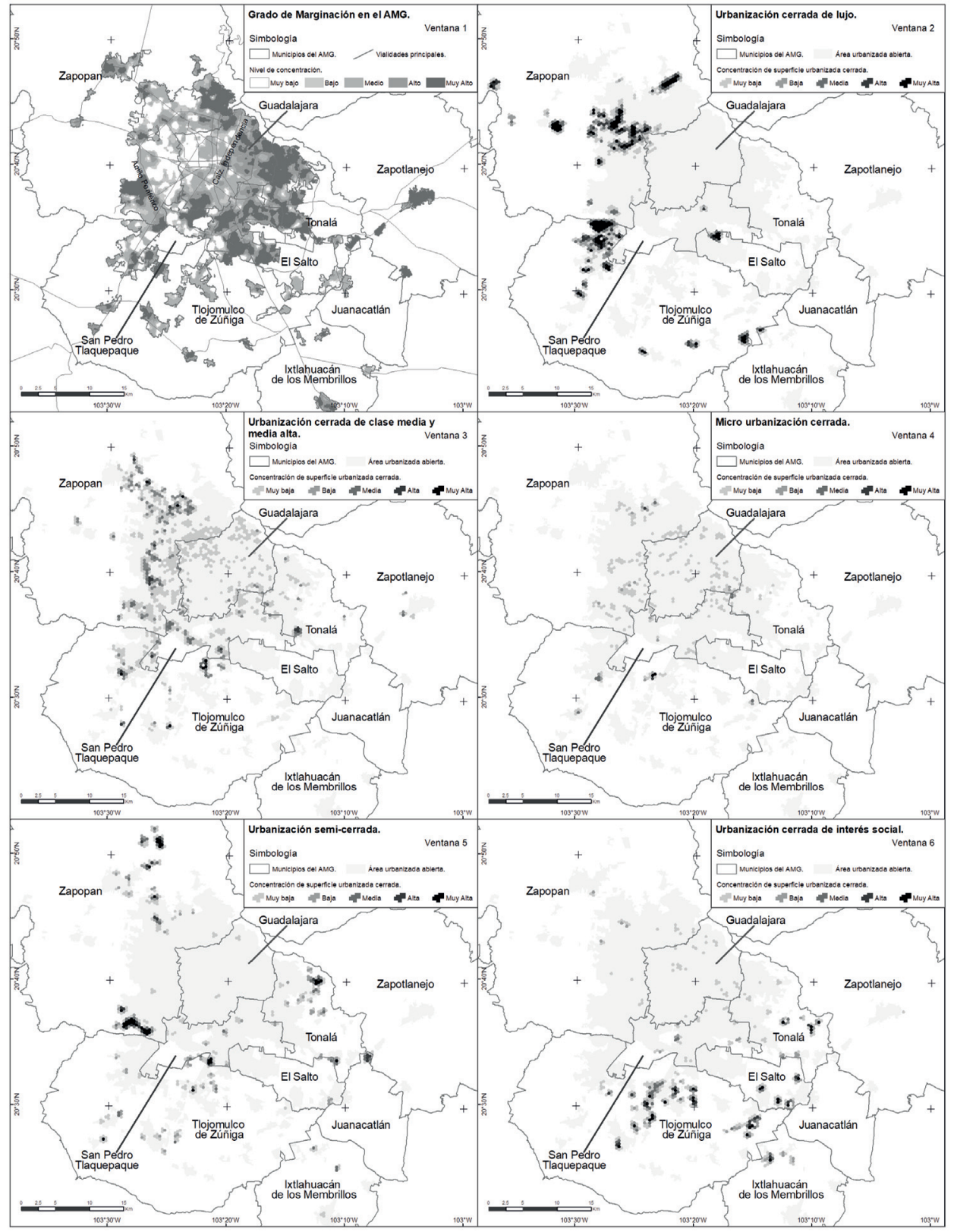

Fuente: Elaboración propia. 
Para identificar la manera en que se distribuye territorialmente la desigualdad social y cómo se relaciona con las urbanizaciones cerradas se ha empleado el Índice de Marginación, una herramienta diseñada por el Consejo Nacional de Población (2010) que, a partir de indicadores como el acceso a educación, salud, características de vivienda y bienes inmuebles, permite determinar cuáles sectores de la ciudad tienen menores oportunidades para mejorar su nivel de vida. La base de datos construida a partir del Censo Nacional de Población y Vivienda 2010 (INEGI, 2010) -Ventana 1- corrobora la tradicional distribución de pobreza en Guadalajara, un fenómeno que fue determinado por la misma geografía de la ciudad desde épocas muy tempranas al propiciar la ubicación de las clases sociales más altas al poniente de la ciudad y relegar a las clases pobres al oriente, siendo el Río San Juan de Dios -hoy Calzada Independencia- el límite entre ambos sectores (Núñez, 2007).

La comparativa entre el nivel de concentración del grado de marginación y la base georreferenciada de UC permite apreciar los resultados del crecimiento urbano. Uno de ellos es la incorporación de las localidades rurales que se encontraban en los diversos municipios integrantes y que, en el caso de Zapopan, terminaron convirtiéndose en pequeñas islas de alto grado de desigualdad que comparten el vecindario con las urbanizaciones cerradas de mayor exclusividad de la ciudad -Ventana 2-.

Las urbanizaciones cerradas dirigidas a clases medias y altas tienen una clara tendencia a mantenerse cerca de los fraccionamientos de lujo y así poder aprovechar la plusvalía que generan -Ventana 3-, mientras que los fraccionamientos de interés social, se concentraron en la parte este y sureste del área metropolitana -Ventana 6-, prosiguiendo con la expulsión de "los no deseados" que busca la ideología neoliberal con el objetivo de crear condiciones que permitan la gentrificación general de la ciudad (Hidalgo y Janoschka, 2014: 17).

En el caso de las micro urbanizaciones cerradas -Ventana 4- pareciera no haber algún patrón particular, pero es posible apreciar una mayor cantidad de ellos en los espacios consolidados de la urbe con una cierta propensión al municipio central. Por su parte los fraccionamientos semi-cerrados -Ventana 5- se presentan como la antítesis de las micro urbanizaciones cerradas, no solo por su ubicación periférica, sino por el cierto grado de "apertura" que tienen hacia la ciudad.

Este par de tipologías son elementos bastante interesantes dentro del proceso de fragmentación socio-espacial ya que operan en los extremos opuestos de la problemática a pesar de contar con ejemplos que pueden llevar a nuevas agrupaciones de análisis; relación entre urbanizaciones de lujo que han abierto las calles y los espacios de alto valor económico que prefirieron encerrarse al adoptar la estructura de la micro urbanización cerrada, densidades de vivienda de espacios semi-abiertos según su nivel socioeconómico, calidad de equipamientos disponibles por tipología, etc.

\section{A modo de conclusión}

Es indiscutible cómo la "neoliberalización urbana" (Hidalgo y Janoschka, 2014: 15) vivida durante las últimas décadas a escala global trajo un reordenamiento de los mercados inmobiliarios en los distintos países latinoamericanos; un contexto en el cual surgió la urbanización cerrada como un producto de inversión y especulación creado a partir de la financiarización de la ciudad. 
La constante evolución del territorio y los cambios sociales experimentados tuvieron como consecuencia que este modelo de vivienda que era dirigido a las élites, cambiara poco a poco para dar cabida a sectores de la población que anteriormente eran desplazados, incrementándose así, la brecha social como parte de una combinación entre el despliegue del capitalismo global y retrocesos significativos en la segregación (Sabatini y Braini, 2008).

El auto-aislamiento de quien vive dentro de la urbanización cerrada y el aislamiento forzado que sufre el ciudadano que habita en la ciudad abierta, o como lo señala Roitman (2004) desde la perspectiva estructuralista de Giddens, segregación voluntaria-involuntaria, fortalece el contraste social del Área Metropolitana de Guadalajara que, según datos del año 2010, se puede estimar un $56 \%$ de población que vive en condiciones que dificultan su acceso a oportunidades para mejorar su calidad de vida, situación que habrá que monitorear constantemente, sobre todo por los cambios que pudo ocasionar el crecimiento expansivo de las urbanizaciones cerradas y la privatización de equipamientos y servicios que ello implica.

Exclusión y fragmentación socio-territorial son vistos como los resultados más nocivos que provoca la urbanización cerrada, y si bien es cierto que aún son factores constantes en cualquier tipología del fraccionamiento cerrado por la presencia de una barrera física, las diferenciaciones que han sido detectadas en las diferentes ciudades de Latinoamérica como parte de la diversificación del modelo destacan la pertinencia de trabajar un enfoque distinto para el estudio de los casos particulares de una ciudad pues, como en el caso de Guadalajara, las relaciones que surgen entre la urbanización cerrada y el entorno inmediato pueden ser tan diversas como sus características.

Por un lado tenemos el ejemplo de la zona residencial de Bugambilias, un fraccionamiento semi-cerrado de lujo que encuentra muy cerca del pueblo de Santa Ana Tepetitlán, localidad que es considerada como zona de alto nivel delictivo (H. Ayuntamiento de Zapopan, 2012-2015); por el otro, se puede considerar a casi cualquiera de los fraccionamientos ubicados al oriente de la metrópoli, conjuntos de alta densidad de vivienda que fueron construidos en áreas marginadas y que son ofrecidos a población de bajos recursos económicos mediante el esquema de vivienda de interés social. Ambos son espacios que han sido identificados por la sociedad como urbanizaciones cerradas debido a su capacidad compartida de fragmentar el territorio, pero más allá de esta similitud, sus diferencias son considerables; desde fraccionamientos que están integrados por residencias valuadas en millones de pesos, gran cantidad de espacios verdes y servicios de alta calidad; hasta aquellos fraccionamientos donde las casas han sido construidas de manera deficiente, los espacios se encuentran abandonados y tienen un alto grado de deterioro.

Aunque no ha sido la mejor manera para afrontar las problemáticas urbanas, la población que está optando por la vida fortificada percibe una mejoría en su estilo de vida ya que se ha resuelto en cierta medida la incapacidad del Estado para cubrir necesidades básicas, desafortunadamente, la imposición del modelo de urbanización cerrada por parte del sector inmobiliario está generando más dificultades que beneficios por la presencia de un muro entre las personas y la ciudad. En un intento por acaparar un mayor mercado con el argumento de "brindar seguridad", la sociedad se segmenta cada vez más, incrementa la distancia entre ricos y pobres y surgen nuevos estratos sociales pues, lo que comenzó como un intento por atraer nuevos compradores del "estilo de vida" de la urbanización cerrada, terminó conformando una clase baja auto-segregada y otra que continúa siendo forzada a la segregación. 
En un inicio se bosquejó a la urbanización cerrada como un ente que por sí mismo no significaba un problema debido a que las contrariedades que suelen relacionarse con este tipo de ocupación territorial se apreciaban como un producto de la sobre explotación del modelo y su alto consumo de superficie, pero a partir de los resultados preliminares obtenidos mediante la clasificación de los fraccionamientos cerrados, se debe replantear la premisa y contemplar la estratificación de las urbanizaciones cerradas como una consecuencia surgida del mismo concepto que no solo implica una diferenciación entre el afuera y el adentro sino al interior de sí mismo, cambiando el paradigma de la urbanización cerrada.

Construir vivienda aislada no es construir ciudad, y construir muros no es hacer comunidad, se requiere diversificar el análisis y dejar de generalizar los efectos que tienen las urbanizaciones cerradas en el territorio como conjunto para enfocarse en encontrar formas eficaces que permitan resolver los conflictos en función de las características estructurales, sociales y espaciales que presenta cada unidad habitacional amurallada.

\section{Referencias bibliográficas}

AGUILAR, A. \& VÁZQUEZ, M. Crecimiento urbano y especialización económica en México: Una caracterización regional de las funciones dominantes. Investigaciones Geográficas, 2000, $\mathrm{N}^{\circ} 42$, p. 87-108.

ARIAS, P. Crisis metropolitana, especialización económica y nuevas relaciones espaciales en México. Espiral. Estudios sobre Estado y Sociedad, 1997, Vol. 10, № 4, p. 143-166.

ARIZAGA, C. Nuevas urbanizaciones cerradas en los noventa: representaciones del suburbio en sectores medios. Buenos Aires: Instituto de Investigaciones Gino Germani, Facultad de Ciencias Sociales, Universidad de Buenos Aires, 2003.

BECERRIL-SÁNCHEZ, T.; MÉNDEZ, J. \& GARROCHO, C. Urbanizaciones cerradas y transformaciones socioespaciales en Metepec, Estado de México. EURE, 2013, Vol. 39, № 117, p. 191-213.

BORSDORF, A. Barrios cerrados en Santiago de Chile, Quito y Lima: tendencias de la segregación socio-espacial en capitales andinas. En: CABRALES, L.F. Latinoamérica: Países abiertos, Ciudades cerradas. Guadalajara: Universidad de Guadalajara / UNESCO, 2002, p. 581-610.

BORSDORF, A. e HIDALGO, R. Formas tempranas de exclusión residencial y el modelo de la ciudad cerrada en América Latina. El caso de Santiago. Revista de Geografía Norte Grande, 2004, N 32 , p. 21-37.

CABRALES, L.F. Estado del conocimiento sobre las urbanizaciones cerradas en Iberoamérica. En: GUTIÉRREZ, O. (coordinador). La ciudad y el miedo. Girona: VII Coloquio de Geografía Urbana, AGE-Universitat de Girona, 2005.

CABRALES, L.F. Tendencias recientes de las urbanizaciones cerradas y polarización residencial en Guadalajara. En: Segregación social del espacio. Nuevos enfoques y patrones emergentes en México y Chile. Guadalajara: PUEC-UNAM, 2006. Disponible en internet: 
http://www.saludambiental.udg.mx/maestria/II_dip_files/Modulo_II/Urbanizaciones\%20Cerradas\%20en\%20Guadalajara.pdf

CABRALES, L.F. y CANOSA, E. Segregación residencial y fragmentación urbana: los fraccionamientos cerrados en Guadalajara. Espiral. Estudios sobre Estado y Sociedad, 2001, № 20 (7), p. 223-253.

CABRALES, L.F. y CANOSA, E. Nuevas formas y viejos valores: urbanizaciones cerradas de lujo en Guadalajara. En: CABRALES, L.F. Latinoamérica: Países abiertos, Ciudades cerradas. Guadalajara: Universidad de Guadalajara / UNESCO, 2002, p. 93-117.

CÁMARA DE DIPUTADOS. LXIII Legislatura. Reforma al Artículo 115 Constitucional: Fortalecimiento Municipal. 2016. Disponible en internet:

http://www.diputados.gob.mx/ cronica57/contenido/cont8/leer5.htm

CAMUS, M. Vivir en el coto. Fraccionamientos cerrados, mujeres y colonialidad. Guadalajara: Universidad de Guadalajara, Centro Universitario de Ciencias Sociales y Humanidades, 2015.

CARBALLO, T. Buenos Aires y urbanización cerrada: nuevas formas de apropiación y fragmentación del espacio urbano. En: CABRALES, L.F. Latinoamérica: Países abiertos, Ciudades cerradas. Guadalajara: Universidad de Guadalajara / UNESCO, 2002, p. 237-260.

CONSEJO NACIONAL DE POBLACIÓN (CONAPO). Índice de marginación por localidad 2010. Colección: Índices sociodemográficos. 2010. Disponible en internet: http://www.conapo .gob.mx / en/CONAPO/Indice_de_Marginacion_por_Localidad_2010

DE LIMA, J. \& RIBEIRO, B. Os condominios horizontais fechados em ciudades médias brasileiras. En: CABRALES, L.F. Latinoamérica: Países abiertos, Ciudades cerradas. Guadalajara: Universidad de Guadalajara / UNESCO, 2002, p. 373-396.

DÍAZ, V. y ORTíZ, A. La ciudad y la arquitectura del miedo. Estudio conceptual comparativo entre el fraccionamiento habitacional cerrado de lujo contemporáneo y la bastida medieval. Revista Nodo, 2014, Vol. 16, Nº 8, p. 25-41.

ENRÍQUEZ, J. Ciudades de muros. Supuestos y claves de entrada para el estudio de las urbanizaciones cerradas. Imaginales. Revista de Investigación Social, 2006, № 4, p. 65-91.

ENRÍQUEZ, J. Socialización y tipología de las urbanizaciones cerradas en Tijuana. Revista Frontera Norte, 2007, Vol. 38, No 19, p. 127-156.

GARCÍA, F. y PERALTA, M. Urbanizaciones cerradas y su vinculación con el espacio exterior en la ciudad de Cali: una propuesta metodológica para su análisis. Prospectiva, 2014, № 19, p. 197-221.

GONZÁLEZ, S. y MEDINA, M. Globalización, funcionalidad económica y estructura urbana en Guadalajara, 1990-2000. En: International Conference Virtual City and Territory. España: Centre de Política de Sòl i Valoracions, 2010. 
H. AYUNTAMIENTO DE ZAPOPAN. Zapopan, estrategia territorial para la prosperidad urbana 2030. México: Administración del Ayuntamiento de Zapopan, 2012-2015.

HIDALGO, R. De los pequeños condominios a la ciudad vallada: las urbanizaciones cerradas y la nueva geografía social en Santiago de Chile (1990-2000). EURE, 2004, Vol. 30, No 91, p. 29-52.

HIDALGO, R.; SALAZAR, A. y ÁLVAREZ, L. Los condominios y urbanizaciones cerradas como nuevo modelo de construcción del espacio residencial en Santiago de Chile (1992-2000). Scripta Nova. Revista Electrónica de Ciencias Sociales, 2003, N 146 (123). Disponible en internet: http://www. ub.edu/geocrit/sn/sn-146(123).htm

HIDALGO, R.; BORSDORF, A.; ZUNINO, H. y ÁLVAREZ, L. Tipologías de expansión metropolitana en Santiago de Chile: Precariópolis estatal y Privatópolis inmobiliaria. Scripta Nova. Revista Electrónica de Ciencias Sociales, 2008, № 270 (113). Disponible en internet: http://www.ub.edu/geocrit/ sn/sn-270/sn-270-113.htm

HIDALGO, R. y JANOSCHKA, M. La ciudad neoliberal: estímulos de reflexión crítica. En: HIDALGO, R. y JANOSCHKA, M. La ciudad neoliberal. Gentrificación y exclusión en Santiago de Chile, Buenos Aires, Ciudad de México y Madrid. Santiago de Chile: Instituto de Geografía, Pontificia Universidad Católica de Chile, 2014, p. 7-32.

ICKX, W. Los fraccionamientos cerrados en la Zona Metropolitana de Guadalajara. En: CABRALES, L.F. Latinoamérica: Países abiertos, Ciudades cerradas. Guadalajara: Universidad de Guadalajara / UNESCO, 2002, p. 117-141.

INSTITUTO METROPOLITANO DE PLANEACIÓN DEL ÁREA METROPOLITANA DE GUADALAJARA (IMEPLAN). Área Metropolitana de Guadalajara. Expansión urbana. Análisis y prospectiva: 19702045. Guadalajara: Editoriales e Industrias Creativas de México, 2015.

INSTITUTO NACIONAL DE GEOGRAFÍA Y ESTADÍSTICA (INEGI). Sistema para la Consulta de Información Censal 2010. Versión 0520012, 2010. Disponible en Internet: http://www.inegi.org.mx/est/ scince/scince2010.aspx

JANOSCHKA, M. El nuevo modelo de la ciudad latinoamericana: fragmentación y privatización del espacio urbano de Buenos Aires. EURE, 2002, Vol. 28, Nº 85, p. 80-117.

LINARES, S. y LAN, D. Análisis multidimensional de la segregación socioespacial en Tandil (Argentina) aplicando SIG. Investigaciones Geográficas, 2007, № 44, p. 149-166.

MALIZIA, M. y PAOLASSO, P. Countries y barrios privados en Yerba Buena, Gran San Miguel de Tucumán, Argentina: nuevas formas de expansión urbana. Estudios Demográficos y Urbanos, 2009, Vol. 24, No 3, p. 583-613.

MARTÍNEZ, H. Geografía e historia. Relación y convergencia epistemológica a través de tres conceptos: Paisaje, región y territorio. Guadalajara: Universidad de Guadalajara, 2001. 
MÉNDEZ, E. y ARIEL, N. La segregación urbana: Una breve revisión teórica para urbanistas. Revista de Arquitectura, 2008, N 10, p. 34-48.

MONTESINO, J. Presencia institucional de las fuerzas armadas en América Latina y su impacto en la calidad de vida de la población. Los casos de Argentina, Colombia, Chile y Perú. Santiago de Chile: Universidad de Arte y Ciencias Sociales, 2010. Disponible en Internet: http://www.eumed. net /tesis-doctorales/2013/jlmj/delincuencia-america-latina.html

NÚÑEZ, B. Ciudad Loma Dorada. Un gran desarrollo habitacional en la Zona Metropolitana de Guadalajara. Guadalajara: Colegio de Jalisco, 2007.

NÚÑEZ, B. Zapopan, Tonalá y Tlajomulco de Zúñiga: disyuntivas habitacionales de la zona conurbada de Guadalajara. Guadalajara: Colegio de Jalisco, 2011.

ORDÓÑEZ, S. Nuevo capitalismo, vías de desarrollo y sistemas nacional-regional de innovación: aproximación a partir del caso de Jalisco (México). En: XIV Seminario Internacional de la RII: Crisis mundial y financiarización: impactos urbanos y territoriales. México: Red Iberoamericana de Investigadores / UANL, 2016.

ORNELAS, J. La ciudad bajo el neoliberalismo. Papeles de Población, 2000, Vol. 23, № 6, p. 45-69.

PÉREZ, F. La consolidación de la segregación espacial desde las prácticas e imaginarios cotidianos de la vida doméstica. Santiago 1930-1960. En: CARMAN, M.; VIEIRA, N. y SEGURA, R. Segregación y diferencia en la ciudad. Quito: FLACSO / CLACSO / Ministerio de Desarrollo Urbano y Vivienda de Ecuador, 2013, p. 197-224.

PRADA, J. Privatización del espacio en los nuevos contextos urbanos y socioculturales: El fenómeno de las Gated Communities. Lurralde: Investigación y espacio, 2011, №34, p. 219-234.

RIWILIS, V. Los barrios cerrados en las Metrópolis Latinoamericanas: ¿Nuevo producto inmobiliario o nueva forma de Habitar la ciudad? En: PEREIRA, P.C.X. e HIDALGO, R. Producción inmobiliaria y reestructuración metropolitana en América Latina. Santiago de Chile: Instituto de Geografía, Pontificia Universidad Católica de Chile, 2008, p.119-135.

RODRÍGUEZ, I. y MOLLÁ, M. La vivienda en las urbanizaciones cerradas de Puebla y Toluca. Scripta Nova. Revista Electrónica de Ciencias Sociales, 2003, № 146 (119). Disponible en internet: http:// www.ub.edu/geocrit/sn/sn-146 (119).htm

ROITMAN, S. Urbanizaciones cerradas: estado de la cuestión hoy y propuesta teórica. Revista de Geografía Norte Grande, 2004, Nº 32, p. 5-19.

ROITMAN, S. Urbanizaciones cerradas a escala planeraria. Prospectiva. Revista de Trabajo Social e Intervención Social, 2016, №21, p. 13-22.

ROMO, C. Las urbanizaciones residenciales cerradas en la periferia de Córdoba. Nuevas modalidades e expansión urbana del siglo XXI. Revista Pensum, 2015, № 1, p. 83-97. 
SABATINI, F. y BRAIN, I. La segregación, los guetos y la integración social urbana: mitos y claves. EURE, 2008, Vol. 34, No 103, p. 5-26.

SABATINI, F.; RASSE, A.; MORA, P. \& BRAIN, I. ¿Es posible la integración residencial en las ciudades chilenas? Disposición de los grupos medios y altos a la integración con grupos de extracción popular. EURE, 2012, Vol. 38, № 115, p. 159-194.

SCHTEINGART, M. La división social del espacio en las ciudades. Perfiles Latinoamericanos, 2001, No 19, p. 13-31.

SOUZA, C. A vida em separado. Estudo de um condominio fechado de Porto Alegre. Porto Alegre: Tesis presentada para obtener el grado en Ciencias Sociales, Universidade Federal do Rio Grande do Sul, 2008. Disponible en internet: http://www.lume.ufrgs.br/bitstream/handle/10183/ 17524/000715370.pdf

THUILLIER, G. El impacto socio-espacial de las urbanizaciones cerradas: el caso de la Región Metropolitana de Buenos Aires. EURE, 2005, Vol. 31, N 93, p. 5-20.

URQUIETA, P. Introducción. En: URQUIETA, P. Ciudades en transformación. Disputas por el espacio, apropiación de la ciudad y prácticas de ciudadanía. La Paz: CIDES-UMSA, 2011, p. 13-28.

VALENZUELA, A. Las nuevas centralidades: fragmentación, espacio público y ciudadanía. En: CABRALES, L.F. Latinoamérica: Países abiertos, Ciudades cerradas. Guadalajara: Universidad de Guadalajara / UNESCO, 2002, p. 31-64.

VIDAL-KOPPMANN, S. Diseño urbano y control del espacio. De la ciudad privada a la ciudad blindada. En: XIII Coloquio Internacional de Geocrítica. El control del espacio y los espacios de control, Universidad de Barcelona, 2014. Disponible en Internet: http://www.ub.edu/ geocrit/coloquio2014/Sonia\%20Vidal-Koppmann.pdf

VILLAR, A. y GARCÍA, M. Ciudad segregada en España: urbanizaciones cerradas en Valencia y Sevilla. Revista INVI, 2016, Vol. 31, № 86, p. 145-177. 